\title{
Development of Emergency Medical Services Amid 5 Years' Experience at a Medical School in Thailand
}

\author{
Korakot Apiratwarakul ${ }^{1}$, Phongphat Ruamsuk ${ }^{1}$, Takaaki Suzuki ${ }^{2}$, Ismet Celebi ${ }^{3}$, Somsak Tiamkao ${ }^{4}$, Vajarabhongsa \\ Bhudhisawasdi ${ }^{1}$, Kamonwon lenghong ${ }^{1 *}$
}

${ }^{1}$ Department of Emergency Medicine, Faculty of Medicine, Khon Kaen University, Khon Kaen, Thailand; ${ }^{2}$ Department of Emergency and Critical Care Medicine, University of Tsukuba Hospital, Tsukuba, Japan; ${ }^{3}$ Department of Paramedic, Gazi University, Ankara, Turkey; ${ }^{4}$ Department of Medicine, Faculty of Medicine, Khon Kaen University, Khon Kaen, Thailand

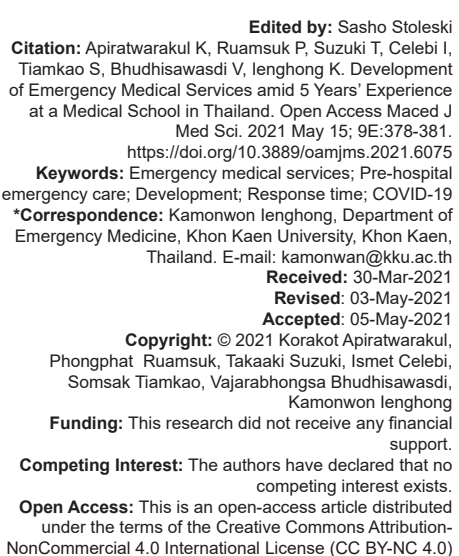

\begin{abstract}
BACKGROUND: The development of emergency medical services (EMSs) in Thailand is divided into two phases following the enactment of the Emergency Medical Act in 2007 aimed at making the work model more systematic. However, the amount of EMS operations has not been well studied.

AIM: The aim of this study was to describe the pattern of EMS operations throughout a 5-year period.

METHODS: A retrospective, single-centered study at a medical school hospital in Thailand. Data were gathered from the EMS database at Srinagarind Hospital throughout the years 2016-2020.

RESULTS: A total of 10,384 EMS operations were examined over a 5-year period (2016-2020). The mean age of patients in 2016 was $40.2 \pm 3.5$ years, and $55.3 \%(n=1178)$ were male. Operations were most commonly performed during the afternoon shift (4.00 p.m.-0.00 a.m.) $41.0 \%, 38.6 \%, 39.5 \%, 39.2 \%$, and $50.8 \%$, respectively. The amount of EMS members had a tendency to increase in number throughout the 5 years of study $(p=0.022)$. The average times from 1669 center call receipt to arrival on scene (response time) for 2016 and 2020 were $8.52 \pm 2.20$ min and $5.52 \pm 3.02 \mathrm{~min}$, respectively $(p<0.001)$
\end{abstract}

CONCLUSION: The development of EMS at Srinagarind Hospital took place with an increase in the age of patients

number of operations in the afternoon shift, and EMS members, yet with a decrease in response times.

\section{Introduction}

The development of emergency medical services (EMSs) in Thailand is divided into two phases, the first period being before 2007. At that stage, there was no specific supervisory agency or law, and most staff worked in the form of volunteers or only for hospitals. Later, problems relating to a lack of human resource management systems, emergency patient assistance equipment, as well as the lack of a responsible agency occurred. Poorly coordinated operations were causing the emergency patients to lose their life or experience organ defects, resulting in unreasonable aggravation of injury or illness. Therefore, the Emergency Medical Act was enacted in 2007. In the second phase of development, the model works more systematically: There are trained personnel according to specific criteria, resulting in greater access to the public through the number 1669 , with free medical services.

The previous studies have shown that modern EMS must have a data link between event data recorders and those within the hospital's electronic health record [1]. By operating EMS under appropriate technology such as telehealth, it can reduce the delivery time of non-emergency patients to the hospital [2], and the development of a systematic database assists to develop an effective service model, for example, the use of Utstein to collect out-of-hospital cardiac arrest patients which has a format that is of international standard. It is possible to compare interagency or international EMS practice [3], [4], [5]. In addition, the timing or date of the operation should be analyzed as these factors influence the operations of EMS [6], [7], [8], [9], [10], [11]. A study in India, the second-most populous country in the world, found that the early development of an EMS should begin with a single aggregate of telephone numbers used for notification in the whole country for the convenience of users [12]. In Saudi Arabia, studies show that one in three people do not know the telephone number to call for EMS operations [13].

As for Srinagarind Hospital, the EMS was established in 2008 with EMS personnel consisting of emergency physicians (EPs) acting as medical directors, emergency nurse practitioners (ENPs), 
advanced emergency medical technicians (AEMTs), and emergency medical technicians (EMTs). The service is divided into two operation levels: An advanced operating unit with a trained doctor specialized in emergency medicine as the head of the operating team and the basic level unit. ENPs act as the head of the operating suite. The service covers an area within Khon Kaen University and neighboring areas with an operating area of approximately $250 \mathrm{~km}^{2}$. Therefore, the collection of operational data to study will be added to the operating model to develop the service in the future to be at its most efficient.

\section{Methods}

\section{Study design and setting}

This was a retrospective, single-centered study at a medical school hospital in Thailand. Data were gathered from the EMS database at Srinagarind Hospital throughout the years 2016-2020. Each case received an operation number from the EMS center and was evaluated. Ethical approval was provided by the Khon Kaen University Ethics Committee for Human Research (HE641174). The requirement for informed consent was waived since confidentiality protection had already been guaranteed. Accordingly, participants were not identified by name, but instead by a unique study number.

\section{Participants}

We included all Srinagarind Hospital's EMS operations between January 1, 2016, and December 31, 2020. Cases with incomplete data and those in which patients were being referred to other hospitals were excluded from the study.

\section{Data collection}

This study employed information from the EMS database of Srinagarind Hospital. Recorded data included information on the number of operations, sex, age, and time spent amid EMS operations. Hospital operating finances were gathered from the financial unit of Srinagarind Hospital. A $1^{\text {st }}$ year trained emergency medicine resident was responsible for data collection under direct supervision of an emergency medicine specialist.

\section{Sample size and statistical analysis}

Sample size was calculated based on the number of EMS operations [14]. To achieve a significance level of $5 \%$ and power of test of 0.80 , we determined that a sample size of 10,384 would be required. Statistical analysis was performed using IBM SPSS for Windows version 26.0, Khon Kaen University license (SPSS Inc., Chicago, IL, USA). Categorical data were presented as percentages, with continuous data presented employing mean and standard deviation. Univariable analysis was carried out using a two sample t-test for numerical data and Pearson's correlation for data relationships between groups.

\section{Results}

A total of 10,384 EMS operations were examined over a 5-year period (2016-2020). The characteristics of subjects and services are shown in Table 1. The mean age of patients in 2016 was $40.2 \pm 3.5$ years, and $55.3 \%(n=1178)$ were male. Operations were most commonly performed during the afternoon shift (4.00 p.m.-0.00 a.m.) 41.0\%, 38.6\%, $39.5 \%, 39.2 \%$, and $50.8 \%$, respectively. EMS members comprised EPs, emergency medicine residents, ENPs, AEMTs, and EMTs which had a tendency to increase in number throughout the 5 years of study ( $p=0.022$ ). Average response times from the 1669 call center receipt to arrival on scene (response time) for 2016 and 2020 were $8.52 \pm 2.20 \mathrm{~min}$ and $5.52 \pm 3.02 \mathrm{~min}$, respectively $(p<0.001)$.

Table 1: Characteristics of subjects and services

\begin{tabular}{lllllll}
\hline Characteristics & $\mathbf{2 0 1 6}$ & $\mathbf{2 0 1 7}$ & $\mathbf{2 0 1 8}$ & $\mathbf{2 0 1 9}$ & $\mathbf{2 0 2 0}$ & $\mathrm{p}$-value \\
\hline Age (years), mean $\pm \mathrm{SD}$ & $40.2 \pm$ & $41.1 \pm$ & $40.5 \pm$ & $43.5 \pm$ & $50.1 \pm$ & $0.010^{*}$ \\
& 3.5 & 4.2 & 5.2 & 4.0 & 9.2 & \\
Gender: Male n, (\%) & 1178 & 1045 & 1043 & 1141 & 1072 & 0.068 \\
& $(55.3)$ & $(52.3)$ & $(50.1)$ & $(51.2)$ & $(55.1)$ & \\
Operation duration (\%) & & & & & & $<0.001^{*}$ \\
$\quad$ Morning shift & 32.1 & 35.1 & 36.2 & 35.2 & 20.1 & \\
$\quad$ Afternoon shift & 41.0 & 38.6 & 39.5 & 39.2 & 50.8 & \\
$\quad$ Night shift & 26.9 & 26.3 & 24.3 & 25.6 & 29.1 & \\
Number of EMS crews (n) & & & & & & $0.022^{*}$ \\
EPs & 8 & 9 & 10 & 11 & 12 & \\
Emergency medicine residents & 16 & 20 & 23 & 25 & 27 & \\
ENPs & 0 & 1 & 2 & 4 & 5 & \\
AEMTs & 1 & 1 & 1 & 2 & 2 & \\
EMTs & 5 & 8 & 11 & 13 & 15 & \\
Response time (min), mean \pm SD & $8.52 \pm$ & $8.41 \pm$ & $8.05 \pm$ & $7.54 \pm$ & $5.52 \pm$ & $<0.001^{*}$ \\
& 2.20 & 2.26 & 2.25 & 2.20 & 3.02 & \\
\hline *Statistical significance; SD: Standard deviation; EMS: Emergency medical service; EPs: Emergency \\
physicians; ENPs: Emergency nurse emergency nurse practitioners; AEMTs: Advanced emergency medical \\
technicians; EMTs: Emergency medical technicians; min: Minute
\end{tabular}

The amount of advanced level EMS operations was $673,770,868,949$, and 1518 throughout the 5-year study period, respectively (Figure 1; $p<0.001$ ).

A total number of EMS operations were 2131, 1999, 2081, 2228, and 1945 throughout the 5-year study period, respectively (Figure $2 ; p<0.001$ ).

Total income resultant of EMS operations was $1,210,750,1,172,500,1,244,100,1,347,750$, and $1,362,800$ THB during the 5-year study period, respectively (Figure 3 ). 


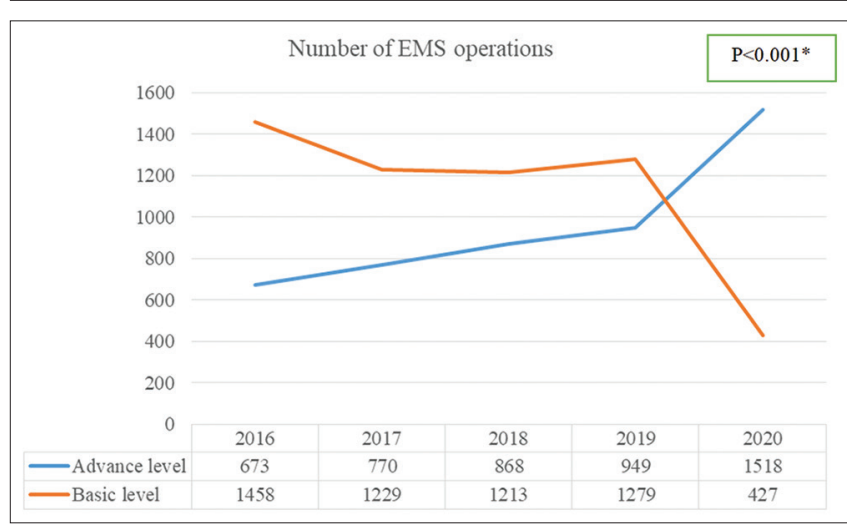

Figure 1: Number of EMS operations (advanced and basic level). *Statistical significance; EMS: Emergency medical service

\section{Discussion}

The present study examined the development of EMS at Srinagarind Hospital throughout a period of 5 years (2016-2020). Most emergency patients were found to be around 40 years of age as per the previous studies [14], [15], [16] except in 2020 when the age of emergency patients utilizing EMS increased as a result of an outbreak of COVID-19 in Thailand and Khon Kaen Province. This caused a decrease in visits to hospitals resulting in a lack of medicines to treat underlying diseases which tended to display more severe symptoms and therefore require more EMS staff to treat patients in this age group.

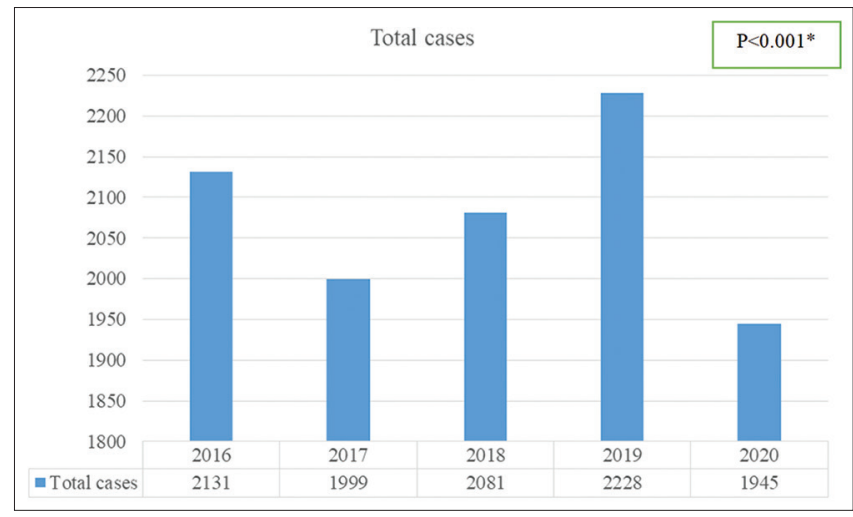

Figure 2: Total number of EMS operations. *Statistical significance, EMS: Emergency medical service

As for EMS operating times, most occurred during the afternoon shift similar to the previous studies [14], [17], [18]. Yet by 2020, there was an increase in period time. This may be due to EMS operations involving bringing in patients with suspected COVID-19 to the hospital; most of which were examined in the afternoon to reduce exposure to patients visiting regular clinics.

Regarding the number of EMS members, it was discovered that there was an increase in both AEMTs, EMTs, doctors, and nurses as a result of the agency's personnel development plan. Since the EMS of Srinagarind Hospital had no paramedic, this limited operations. Therefore, there is planning to develop personnel at all levels to encourage greater potential to enhance the effectiveness of services provided through the direct and indirect medical oversight of EPs.

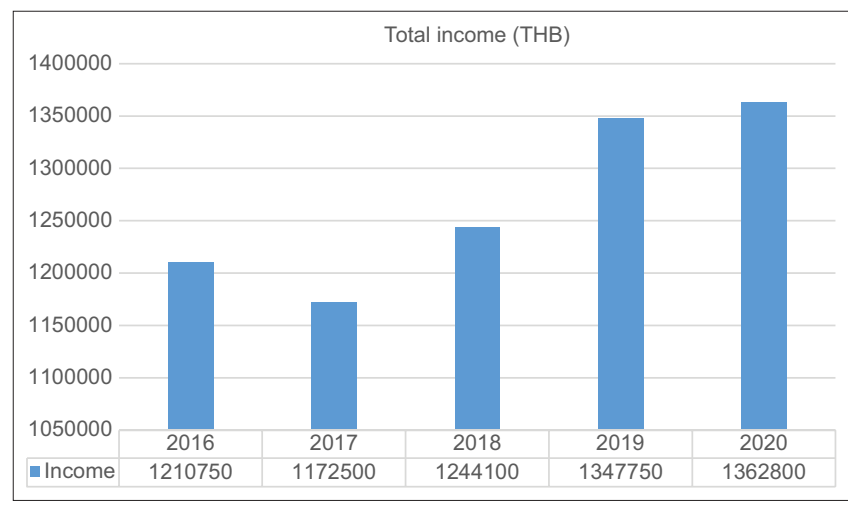

Figure 3: Total income of EMS. THB: Thai baht

In terms of response time, it was found that according to Thailand's National Institute for Emergency Medicine (NIEMs) standard of $8 \mathrm{~min}$, most response times fell within this time frame or were close; especially in 2020, when response times were lower than every other year. This could be due to the COVID19 epidemic which decreased the number of vehicles on the road, making it even faster for ambulances to reach the scene.

As for operational level, most of the operations were at the basic level with the exception of 2020 where advanced level operations increased. This could be an outcome of the COVID-19 epidemic, resulting in the majority of patients not receiving drugs at the hospital or wanting to travel to the hospital. The severity of diseases increases until the application of EMS services, with more severe symptoms resulting in the implementation of operations at an advanced level.

Moreover, the income from compensation for each operation according to NIEMs requirements, at the basic level is $500 \mathrm{THB} /$ case and for advanced level $1000 \mathrm{THB} / \mathrm{case}$, corresponding to the level of operations in 2020. Although there was a decrease in the amount of operations issued, most operations were at an advanced level, resulting in a greater operating income.

However, this study is limited. Since data were obtained from a single EMS center, data integrity may not be referenced by other agencies [14], [15], [16], [17], [18].

\section{Conclusion}

The development and utilization of EMS at Srinagarind Hospital increased in accordance with patient age, number of operations in the afternoon shift, and EMS members. Yet, response times decreased. 


\section{Acknowledgments}

The authors would like to thank all participants in this study as well as Mr. Ross Lacey for acting as an English consultant.

\section{References}

1. Le HP, Hackel S, Guenther A, Goldschmidt R, Daoud M, Deserno TM. International standard accident number: A master case index linking accident and emergency with medical data. Stud Health Technol Inform. 2019;258:120-4.

PMid:30942727

2. Langabeer JR $2^{\text {nd }}$, Gonzalez M, Alqusairi D, ChampagneLangabeer $\mathrm{T}$, Jackson A, Mikhail J, et al. Telehealthenabled emergency medical services program reduces ambulance transport to urban emergency departments. West J Emerg Med. 2016;17(6):713-20. https://doi.org/10.5811/ westjem.2016.8.30660

PMid:27833678

3. Shin J, Chocron R, Rea T, Kudenchuk P, McNally B, Eisenberg M. Merits of expanding the Utstein case definition for out of hospital cardiac arrest. Resuscitation. 2021;158:88-93. https://doi.org/10.1016/j.resuscitation.2020.10.041

PMid:33220350

4. Keykaleh MS, Sohrabizadeh S. The emergency medical system (EMS) response to Iraqi pilgrims' bus crash in Iran: A case report. BMC Emerg Med. 2019;19(1):38. https://doi.org/10.1186/ s12873-019-0253-2

PMid:31311494

5. Goodwin G, Picache D, Gaeto N, Louie BJ, Zeid T, Aung PP, et al. Gender disparities in out-of-hospital cardiac arrests. Cureus. 2018;10(8):e3233. https://doi.org/10.7759/cureus.3233 PMid:30410839

6. Cantwell K, Morgans A, Smith K, Livingston M, Spelman T, Dietze P. Time of day and day of week trends in EMS demand. Prehosp Emerg Care. 2015;19(3):425-31. https://doi.org/10.310 9/10903127.2014.995843

PMid:25664379

7. Modi PD, Solanki R, Nagdev TS, Yadav PD, Bharucha NK, Desai $A$, et al. Public awareness of the emergency medical services in Maharashtra, India: A questionnaire-based survey. Cureus. 2018;10(9):e3309. https://doi.org/10.7759/cureus.3309 PMid:30456003

8. Sasaki S, Comber AJ, Suzuki H, Brunsdon C. Using genetic algorithms to optimise current and future health planning--the example of ambulance locations. Int J Health Geogr. 2010;9:4. https://doi.org/10.1186/1476-072x-9-4

PMid:20109172

9. Tärnqvist J, Dahlén E, Norberg G, Magnusson C, Herlitz J, Strömsöe A, et al. On-scene and final assessments and their interrelationship among patients who use the EMS on multiple occasions. Prehosp Disaster Med. 2017;32(5):528-35. https:// doi.org/10.1017/s1049023x17006458

PMid:28478788

10. Ng WM, De Souza CR, Pek PP, Shahidah N, Ng YY, Arulanandam S, et al. myResponder smartphone application to crowdsource basic life support for out-of-hospital cardiac arrest: The Singapore experience. Prehosp Emerg Care. 2020;24:1-9. https://doi.org/10.1080/10903127.2020.1777233 PMid:32497484

11. Wimalaratne $\mathrm{K}$, Lee $\mathrm{Jl}$, Lee $\mathrm{KH}$, Lee HY, Lee JH, Kang IH. Emergency medical service systems in Sri Lanka: Problems of the past, challenges of the future. Int $\mathrm{J}$ Emerg Med. 2017;10(1):10. https://doi.org/10.1186/s12245-017-0127-x PMid:28224347

12. Sharma M, Brandler ES. Emergency medical services in India: The present and future. Prehosp Disaster Med. 2014;29(3):30710. https://doi.org/10.1017/s1049023×14000296 PMid:24721137

13. EMSAT: The Emergency Medical Service Assessment Team Group, Hamam AF, Bagis MH, AlJohani K, Tashkandi AH. Public awareness of the EMS system in Western Saudi Arabia: Identifying the weakest link. Int J Emerg Med. 2015;8(1):70. https://doi.org/10.1186/s12245-015-0070-7 PMid:26347359

14. Apiratwarakul K, lenghong K, Gaysonsiri D, Mitsungnern T, Buranasakda M, Bhudhisawasdi V. The effectiveness of oxygen-powered inhalation devices in Prehospital care. J Med Assoc Thai. 2020;103 Suppl 6:58-60.

15. Apiratwarakul K, lenghong K, Bhudhisawasdi V, Gaysonsiri D, Tiamkao $\mathrm{S}$. Response times of motorcycle ambulances during the COVID-19 pandemic. Open Access Maced J Med Sci. 2020;8(T1):526-9. https://doi.org/10.3889/oamjms.2020.5527

16. Apiratwarakul K, Songserm $\mathrm{W}$, lenghong $\mathrm{K}$, Phungoen $\mathrm{P}$, Gaysonsiri D, Bhudhisawasdi V. The role of mechanical cardiopulmonary resuscitation devices in emergency medical services. J Med Assoc Thai. 2020;103 Suppl 6:98-101.

17. lenghong $K$, Srikumpa $P$, Apiratwarakul $K$, Phungoen $P$, Gaysonsiri D, Bhudhisawasdi V. Factors associated with transfusion of uncross-matched type-O packed red cells for acute upper gastrointestinal hemorrhage. J Med Assoc Thai. 2020;103 Suppl 6:22-6.

18. lenghong K, Kleebbuakwan K, Apiratwarakul K, Phungoen $\mathrm{P}$, Gaysonsiri D, Bhudhisawasdi V. Comparison of cleaning methods for ultrasound probes at an emergency department in a resource-limited country. J Med Assoc Thai. 2020;103 Suppl 6:67-71. 